\title{
Nuclear Fission: from more phenomenology and adjusted parameters to more fundamental theory and increased predictive power
}

\author{
Aurel Bulgac ${ }^{1, \star}$, Shi Jin ${ }^{1}$, Piotr Magierski ${ }^{2,1}$, Kenneth Roche ${ }^{3,1}$, Nicolas Schunck ${ }^{4}$, and Ionel Stetcu ${ }^{5}$ \\ ${ }^{1}$ Department of Physics, University of Washington, Seattle, WA 98195-1560, USA \\ ${ }^{2}$ Faculty of Physics, Warsaw University of Technology, ulica Koszykowa 75, 00-662 Warsaw, POLAND \\ ${ }^{3}$ Pacific Northwest National Laboratory, Richland, WA 99352, USA \\ ${ }^{4}$ Nuclear and Chemical Science Division, Lawrence Livermore National Laboratory, Livermore, CA 94551, USA \\ ${ }^{5}$ Theoretical Division, Los Alamos National Laboratory, Los Alamos, NM 87545, USA
}

\begin{abstract}
Two major recent developments in theory and computational resources created the favorable conditions for achieving a microscopic description of fission dynamics in classically allowed regions of the collective potential energy surface, almost eighty years after its discovery in 1939 by Hahn and Strassmann [1]. The first major development was in theory, the extension of the Time-Dependent Density Functional Theory (TDDFT) [2-5] to superfluid fermion systems [6]. The second development was in computing, the emergence of powerful enough supercomputers capable of solving the complex systems of equations describing the time evolution in three dimensions without any restrictions of hundreds of strongly interacting nucleons. Thus the conditions have been created to renounce phenomenological models and incomplete microscopic treatments with uncontrollable approximations and/or assumptions in the description of the complex dynamics of fission. Even though the available nuclear energy density functionals (NEDFs) are phenomenological still, their accuracy is improving steadily and the prospects of being able to perform calculations of the nuclear fission dynamics and to predict many properties of the fission fragments, otherwise not possible to extract from experiments.
\end{abstract}

Meitner and Frisch [7] and Bohr and Wheeler [8,9] interpreted the neutron induced fission of uranium observed by Hahn and Strassmann [1] as the Coulomb-driven division of a classically charged liquid drop in competition with the surface tension of the liquid drop and they obtained a good back-of-the-envelope estimate of the total kinetic energy (TKE) of the fission fragments (FFs). Unlike the $\alpha$-decay and spontaneous fission observed later [10], induced fission occurs entirely above the barrier, and therefore is to a large extent a classical process. The impinging neutron on uranium leads to the formation of an excited state, the compound nucleus, in a region of the nuclear spectrum where the level density is extremely high and the nucleus is thus relatively hot. The nucleus evolves for a very long time from its ground state shape towards the top of the outer fission barrier, where the Coulomb repulsion overcomes the surface tension and the nucleus starts its descent to scission, where the two FFs are formed. The top of the fission barrier is energetically barely below the neutron threshold. Although the nucleus is already quite elongated at this point, it has relatively little intrinsic excitation energy and is relatively cold. It was soon realized that in nuclei nucleons form shells, nucleons live on quantized orbits $[11,12]$, and the spin-orbit interaction plays a critical role in the formation of the nuclear shells and ultimately in the formation of the FFs [13]. Hill

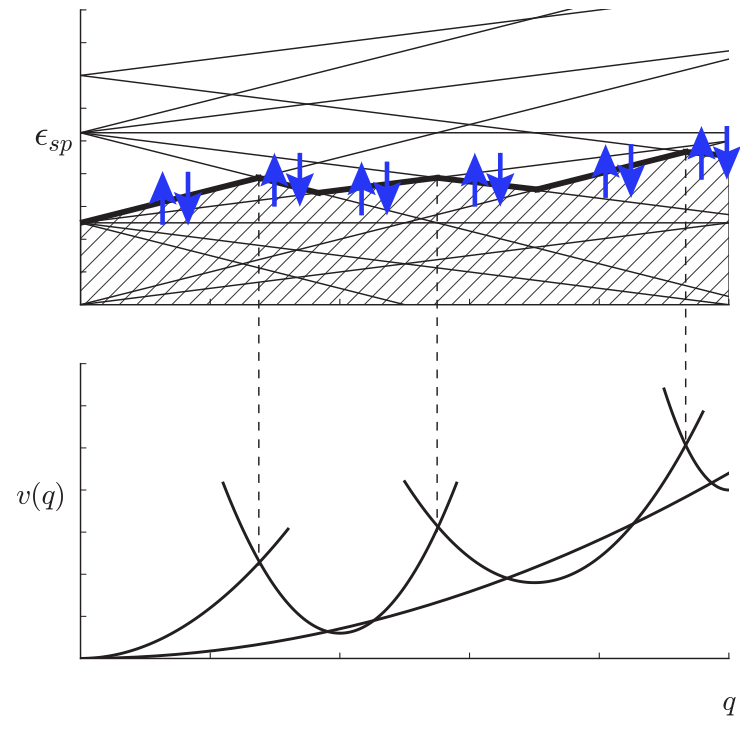

Figure 1. The qualitative evolution of the single-particle levels (upper panel) and of the total nuclear energy (lower panel) as a function of nuclear deformation $[14,15]$. The Fermi level is shown with a thick line.

\footnotetext{
^e-mail: bulgac@uw.edu
} 
and Wheeler [14] discussed how the liquid drop deformation energy emerges from a quantum mechanical approach based on considering the quantized single-particle motion of nucleons in a slowly deforming potential well. In their approach, the liquid drop potential deformation energy was, in the first approximation, an envelope of many intersecting parabolas caused by single-particle level crossings, see Figure 1. At single-particle level crossings of the last occupied level, nucleons jump from one level to another, in order to maintain the sphericity of the Fermi sphere. Without such a redistribution of nucleons at the Fermi level, the Fermi sphere would become oblate when a nucleus deforms on the way to scission into two fragments, while the spatial shape of the nucleus would become ever more prolate. This would lead to a volume excitation energy of the nucleus. This is inconsistent with the fact that nuclei are saturating systems with a large surface tension that can only deform by changing the shape of their surface, while maintaining their volume constant. Hence, only the Coulomb and the surface contributions to the total energy change. Each single-particle level is typically double degenerate, due to the Kramers degeneracy, and nucleons have to jump in pairs, otherwise the nuclear shape evolution towards scission would be hindered $[15,16]$. In this case, nucleons would follow diabatic levels instead of adiabatic ones, as was indeed indirectly established in recent TDHF simulations of fission dynamics [17, 18]. Pairing correlations, while relatively weak in nuclei, are very effective at promoting simultaneously two nucleons from time-reversed orbitals into other time-reversed orbitals and thus they greatly facilitate the evolution of the nuclear shape towards scission. An approximate treatment of the pairing correlations in fission was implemented using either a frozen [19] or time-dependent [20] BCS approximation in conjunction within the TDHF approach, but only at relatively large elongations, where FFs were practically formed. It was established in the 1960's and 1970's that single-particle level bunching exists in nuclear systems not only in the case of spherical nuclei (as in the case of atoms), but also in deformed and highly deformed nuclei. At first this phenomenon was experimentally observed in the case of fission isomers at very large elongations [21$23]$ and subsequently in the case of superdeformed nuclei [24]. The existence of nucleonic shells at large deformations results in a potential energy deformation surface with local maxima and minima, which are otherwise absent in the case of a classical charged liquid drop. The aforementioned level crossings cause discontinuities in the potential energy surface. Some of these discontinuities can be smoothed out when pairing correlations are present, leading to avoided level crossings. Overall fission dynamics is a very complex process, which still did not reach a fully microscopic description [25] in spite of almost eight decades of effort. The formation and the properties of FFs was and still is the most debated and arguably the central topic in phenomenology of nuclear fission. In the absence of a microscopic description and because of the enormous challenges arising from solving the necessarily time-dependent coupled nonlinear Schrödinger equations for hundreds of strongly interacting nucleons, many phe- nomenological models, both quantum and classical in nature, have been suggested over the years. Unfortunately most, if not all, of them lack a solid microscopic underpinning and for that reason their predictive power is limited.

The bulk behavior of the deformation potential energy surface is determined by the surface tension and Coulomb energy, which are rather well-known and understood, and their competition is the main driving force leading to fission as in liquid drop models. The underlying shell structure imprints hills and valleys on the overall charged liquid drop energy landscape $[22,23]$. On the way to scission configuration, nucleons have to go through a large number of redistributions at the single-particle levels crossing around the Fermi level, in order to maintain the spherical symmetry of their local momentum distribution. Overall, the deformation potential energy surface acquires a profile somewhat similar to that of an uneven mountain, with little hills and valleys and covered by trees, and the evolution of the nuclear shape is in the end similar to the erratic motion of a pinball, not straight down the hill, but rather left and right, bouncing (mostly elastically) from the many obstacles on the way to the bottom of the valley, where the pinball eventually breaks up. In the last stages of this complex evolution, the independent character of the nucleons inside the nucleus plays again a critical role, since magic closed shells control the nuclear shape evolution and the FF formation.

Since fission dynamics is a truly non-stationary phenomenon, an extension of the TDDFT [2-5] in the Local Density Approximation (LDA) implementation of Kohn and Sham [3] was needed, and the Superfluid Local Density Approximation (SLDA) [6] was developed and validated quite extensively against experiment and theoretical results obtained using the Schrödinger description. In the case of many strongly interacting particles the Schrödinger equation for a many-body system has to be replaced with the TDDFT, which is mathematically equivalent to the Schrödinger equation for one-body observables and the only theoretical framework suitable to describe the structure and dynamics of quantum many-body systems, if the corresponding energy density functional (the equivalent of the potential in the Schrödinger equation) is known in sufficient detail. TDSLDA allows the evaluation of FFs properties, which are otherwise impossible to either measure or compute, such as FFs excitation energies immediately after scission.

As our simulations within TDSLDA demonstrate [26], see Figure 2, the nucleus separates typically into two unequal FFs. In our calculations, we used in the particle-hole channel a phenomenological NEDF [27], complemented with pairing correlations described as in Refs. [28, 29]. The larger FF, which has properties very similar to the double-magic ${ }^{132} \mathrm{Sn}$, emerges with an almost spherical shape, while the lighter fragment emerges in an elongated configuration, with a ratio of the major to minor axes close to $3 / 2$. To much of our surprise, the properties of the FFs in these simulations [26] are very close to experimental measurements, even though no effort was put into reproducing observations. We attribute this to the fact that the NEDF reproduces bulk nuclear properties relatively well. 
${ }^{240} \mathrm{Pu}$ fission with the normal pairing gap
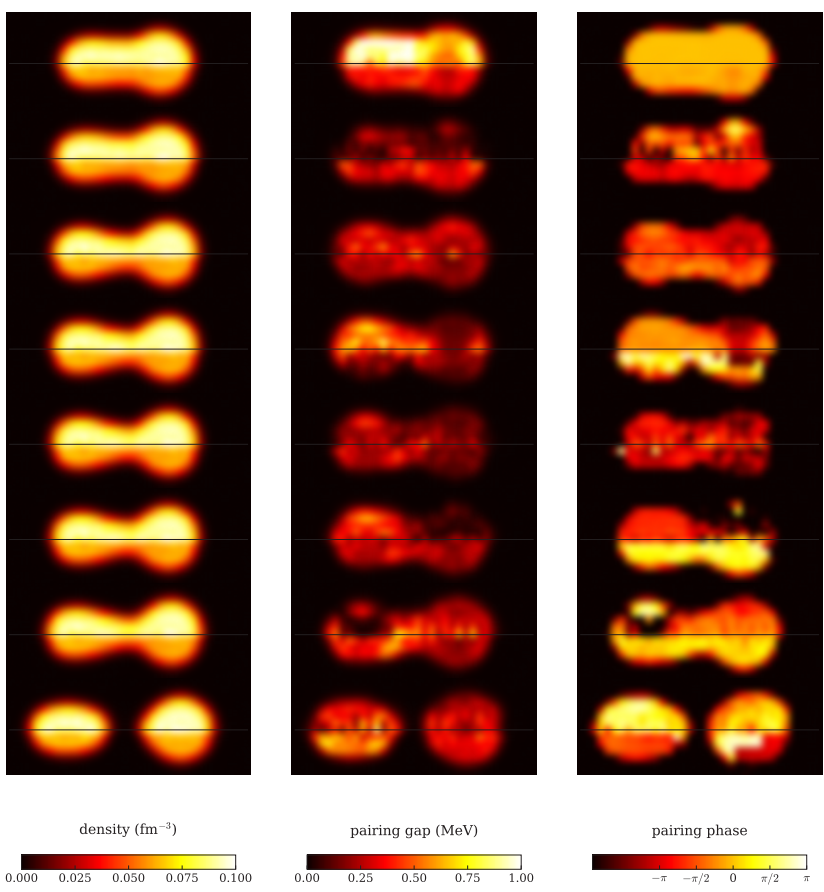

pairing gap (MeV)

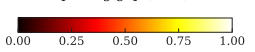

pairing phase

${ }^{240} \mathrm{Pu}$ fission with a larger pairing gap
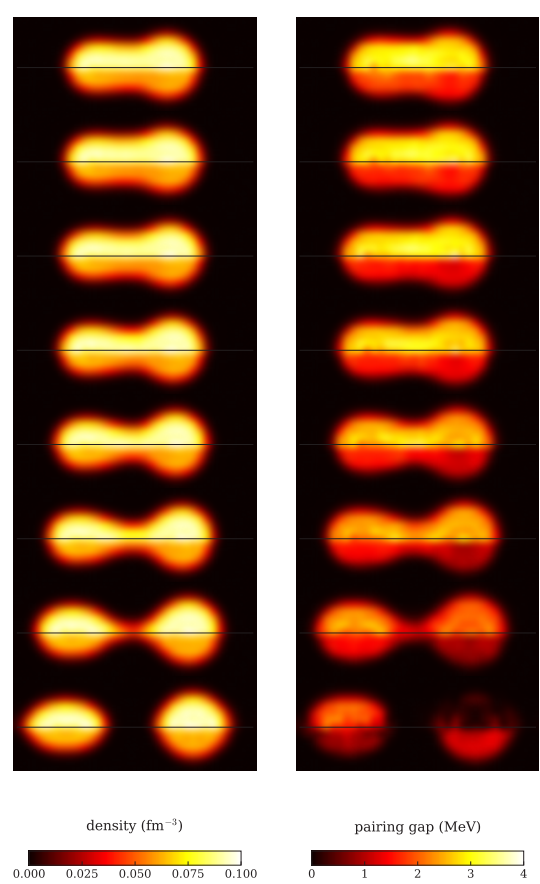

pairing gap (MeV)

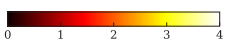

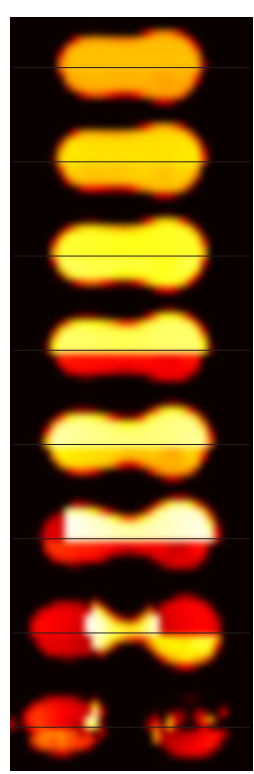

pairing phase

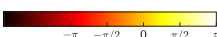

Figure 2. In the upper frames is the induced fission of ${ }^{240} \mathrm{Pu}$ with normal pairing strength last up to $14,000 \mathrm{fm} / \mathrm{c}\left(\approx 47 \times 10^{-21} \mathrm{~s}\right)$ from saddle-to-scission. The columns show sequential frames of the density (1st column), the magnitude of the pairing field ( 2 nd column), and the phase of the pairing field (3rd column). In each frame the upper/lower part of each frame shows the neutron/proton density, the magnitude of neutron/proton pairing fields, and of the phase of the pairing field respectively [26]. In the lower frames is the induced fission of ${ }^{240} \mathrm{Pu}$ with enhanced pairing strength lasts about $1,400 \mathrm{fm} / \mathrm{c}$.

The biggest surprise, however, was the observation that the time from saddle-to-scission was about an order of magnitude larger than ever estimated previously in the literature. This was ulteriorly confirmed by independent simulations [31]. The lower frames of Figure 2 serves as an illustration of the crucial role played by pairing correlations in fission dynamics. In the upper frames one can see that the pairing field on the way from saddle-to-scission does fluctuate noticeably in magnitude and phase. Therefore, strictly speaking the pairing field during its time evolution stops being a superfluid condensate of Cooper pairs, which otherwise would show a uniform phase. By increasing artificially the strength of the pairing field, we observe that fission dynamics proceeds approximatelyt ten times faster, see Figure 2. In that case, the pairing field shows the expected characteristics of a slowly-evolving superfluid condensate, the nuclear fluid behaving almost like a perfect or ideal fluid, where dissipation is practically absent, and the two FFs emerge macroscopically entangled now [30].

At FUSION17 workshop W. Mittig [32] discussed the very intriguing possibility of the existence of long-lived nuclear systems with very large charges, $Z \approx 200$ or even higher. One can contemplate the collision of two heavy nuclei with an energy just at the Coulomb barrier, when fusion is likely to occur. As in the case of fission dynamics, immediately after fusion many collective degrees of freedom of the combined system are likely to get excited, leading to a depletion of the energy from the incoming collision channel. This combined nuclear system might thus survive for a relatively long time, but eventually the system will decay into two almost equal fragments, as the combined system would likely equilibrate, even in the case of asymmetric collisions.

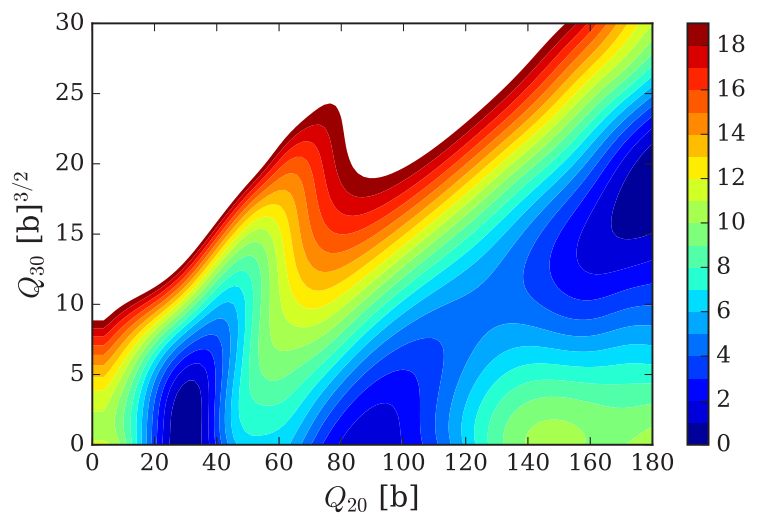

Figure 3. The potential energy surface of ${ }^{240} \mathrm{Pu}$ evaluated with the new energy density functional SeaLL1 [36].

A major limitation of the DFT is a lack of a method to evaluate two-body observables, such as the width of the FFs mass and charge yields and of the total TKE of the FFs. Within the TDHF approach one can use the Balian and Vénéroni (BV) [33] prescription or, alternatively, the more involved stochastic mean-field model introduced by Ayik [34]. Ayik's model is computationally more costly than the TDHF with the BV prescription included. The 
widths estimated using either the BV or the Ayik prescription are mathematically identical under certain conditions [34]. Ayik's prescription has been applied recently to the spontaneous fission of ${ }^{258} \mathrm{Fm}$ [31] in an approach characterized by the authors somewhat optimistically as "the first time a fully microscopic description of the fragment TKE distribution after fission and gives unique microscopic information on the fission process." While appealing, Ayik's model is nevertheless phenomenological in nature (as is random matrix theory), it relies on a several assumptions, and it is prone to large numerical noise. The main difference of the approach of Ref. [31] and TDHF calculations performed in the past is in choosing an ad hoc ensemble of stochastic initial conditions, instead of a single initial nuclear shape, at the point where the nucleus exits the outer fission barrier. In the case of spontaneous fission the nucleus emerges from under the barrier likely in its ground state corresponding to that instantaneous shape with $Q_{20} \approx 100$ barn in case of ${ }^{258} \mathrm{Fm}$ [31]. These authors [31] however chose to start their fission dynamics much further, when $Q_{20}$ is either 125 or 160 barn, at which point the authors impose a thermal excitation of the nucleus to an energy corresponding to the ground state. This stochastic ensemble has large unphysical fluctuations of all possible observables, since most of the single-particle occupation probabilities obtained after diagonalizing the stochastic one-body density matrix $\rho(t)=\sum_{i j} \phi_{i}(t) \rho_{i j} \phi_{j}^{*}(t)$ acquire unphysical values, either with very large negative or very large positive values outside the interval $[0$, 1]. (Here $\rho_{i j}$ are Gaussian random complex numbers.) Ref. [31] and Ref. [20] using the BV-prescription are so far the only attempts to describe quantum mechanically within TDHF the distribution of the FFs mass, charge yields, and TKE distributions. The mass widths using the BV prescription [20] appear visually narrower than those estimated in Ref. [31]. There is no theoretical argument presented in Ref. [31] to choose as a starting point of the dynamical simulations the arbitrary deformations $Q_{20}=$ 125 or 160 barn. It would be more natural to start the simulation at the exact configuration where the nucleus emerged from under the barrier at $Q_{20} \approx 100$ barn. In that case the size of the "quantum fluctuations" would be zero and the nucleus would have no intrinsic excitation energy. But that would deprive the authors from the ability to generate a desired FFs distribution. The authors even establish that if they were to start their simulations closer to the scission configurations their results would be quite different, thus precluding this approach of its predictive power. One can safely conclude that the FFs distributions definitely depend strongly on the choice of the initial conditions within such a stochastic approach. A more natural approach would be a TDGCM approach advocated by Regnier et al. [35], in which one generates the distribution of the initial conditions in a TDDFT simulation of fission dynamics.

The predictive power of a TDDFT approach depends critically on the quality of the NEDFs, which are phenomenological so far and depend on a rather large number of fitting parameters (typically 14 and sometimes even more), some of them rather strongly correlated with each other. This points to the fact that there are elements of NEDF that are still poorly understood and there is a need for better constrained NEDFs, either phenomenologically or microscopically. We have developed a qualitatively new NEDF [36], which contains terms previously not considered in literature, which depends on only 7 uncorrelated parameters, with a rms for 602 masses of even-even nuclei from the AME2012 [37] of $1.74 \mathrm{MeV}$ and for 345 corresponding charge radii of $0.034 \mathrm{fm}$. The potential energy surface for ${ }^{240} \mathrm{Pu}$ predicted by SeaLL1, see Figure 3, has potential energy barriers comparable to those obtained in FRLDM [38], though a relatively low energy of the fission isomer, and a nice single-particle spectrum of ${ }^{208} \mathrm{~Pb}$, see Figure 4.

This work was supported in part by U.S. Department of Energy (DOE) Grant No. DE-FG02-97ER41014 and by the U.S. Department of Energy under Contract Nos. DEAC52-07NA27344 (Lawrence Livermore National Laboratory). This research used resources of the Oak Ridge Leadership Computing Facility, which is a DOE Office of Science User Facility supported under Contract DEAC05-00OR22725. It also used resources of the National Energy Research Scientific Computing Center, a DOE Office of Science User Facility supported by the Office of Science of the U.S. Department of Energy under Contract No. DE-AC02-05CH11231. Some of the calculations were performed on Moonlight at LANL. Computing support for this work also came from the Lawrence Livermore National Laboratory (LLNL) Institutional Computing Grand Challenge program.

\section{References}

[1] O. Hahn and F. Strassmann, Naturwissenschaften 27, 11 (1939).

[2] P. Hohenberg and W. Kohn, Phys. Rev. 136, B864 (1964).

[3] W. Kohn and L.J. Sham, Phys. Rev. 140, A1133 (1965).

[4] R.M. Dreizler and E.K.U. Gross, Density Functional Theory: An Approach to the Quantum Many-Body Problem, (Springer-Verlag, Berlin, 1990).

[5] Fundamentals of Time-Dependent Density Functional Theory, Eds. M.A.L. Marques, et al., Lecture Notes in Physics, vol. 837 (Springer-Verlag, Berlin, 2012).

[6] A. Bulgac, Ann. Rev. Nucl. Part. Sci. 63, 97 (2013).

[7] L. Meitner and O.R. Frisch, Nature (London), 143, 239 (1939).

[8] N. Bohr, Nature (London), 143, 330 (1939).

[9] N. Bohr and J.A. Wheeler, Phys. Rev. 56, 426 (1939).

[10] G.N. Flerov and K.A. Petrzhak, Phys. Rev. 58, 275 (1940).

[11] Maria Goeppert Mayer, Phys. Rev. 75, 1969 (1949).

[12] O. Haxel, J.H.D. Jensen, and H.E. Suess, Phys. Rev. 75, 1766 (1949).

[13] L. Meitner, Nature, 165, 561 (1950).

[14] D.L. Hill and J.A. Wheeler, Phys. Rev. 89, 1102 (1953). 

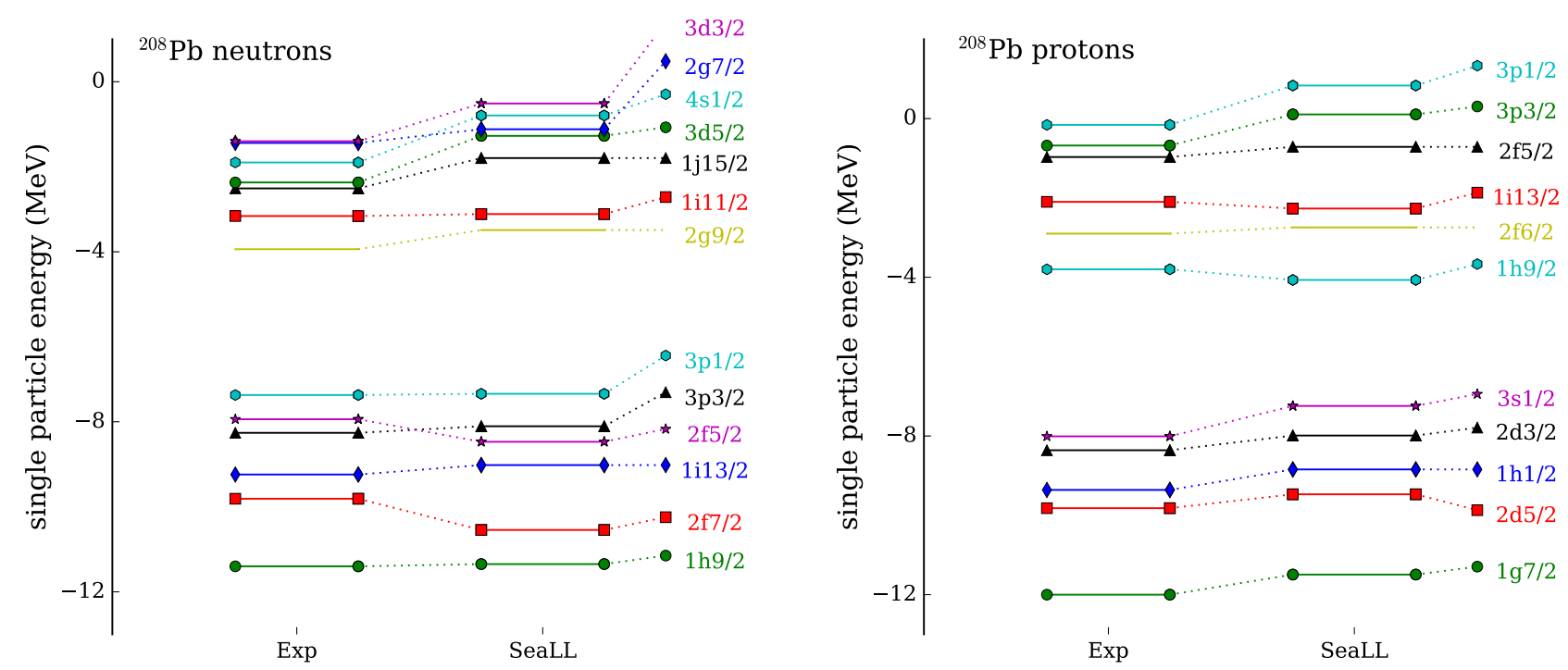

Figure 4. The single-particle energy levels ${ }^{208} \mathrm{~Pb}$ evaluated with the new energy density functional SeaLL1 [36] versus experiement.

[15] G.F. Bertsch, Phys. Lets. B 95, 157 (1980).

[16] G.F. Bertsch and A. Bulgac, Phys. Rev. Lett. 79, 3539 (1997).

[17] P. Goddard, P. Stevenson, and A. Rios, Phys. Rev. C 92, 054610 (2015).

[18] P. Goddard, P. Stevenson, and A. Rios, Phys. Rev. C 93, 014620 (2016).

[19] C. Simenel and A.S. Umar, Phys. Rev. C 89, 031601(R) (2014).

[20] G. Scamps, C. Simenel, and D. Lacroix, Phys. Rev. 92, 011602(R) (2015).

[21] S.M. Polikanov, et al., JETP (Sov. Phys.) 42, 1016 (1962)

[22] M. Brack, et al., Rev. Mod. Phys. 44, 32 (1972).

[23] S. Bjørnholm and J.E. Lynn, Rev. Mod. Phys. 52, 725 (1980).

[24] P.J. Twin, et al., Phys. Rev. Lett. 57, 811 (1986).

[25] N. Schunck and L.M. Robledo, Rep. Prog. Phys. 79, 116301 (2016).

[26] A. Bulgac, P. Magierski, K.J. Roche, and I. Stetcu, Phys. Rev. Lett. 116, 122504 (2016).
[27] E. Chabanat, P. Bonche, P. Haensel, J. Meyer, R. Schaeffer, Nucl. Phys. A635, 231 (1998).

[28] A. Bulgac and Y. Yu, Phys. Rev. Lett. 88, 042504 (2002)

[29] Y. Yu and A. Bulgac, Phys. Rev. Lett. 90, 222501 (2003).

[30] A. Bulgac and S. Jin, an updated version of arXiv:1701.06683 to be resubmitted.

[31] Y. Tanimura, D. Lacroix, and S. Ayik, Phys. Rev. Lett. 118, 152501 (2017).

[32] W. Mittig, talk presented at FUSION17, Hobart, Tasmania, February 20-24, 2017.

[33] R. Balian and M. Vénéroni, Phys. Lett. B136, 301 (1984).

[34] S. Ayik, Phys. Lett. B658, 174 (2008).

[35] D. Regnier, N. Dybray, N. Schunck, and M. Verrière, Phys. Rev. C 93, 054611 (2016).

[36] A. Bulgac. M.M. Forbes, S. Jin, N. Schunck, and R. Perez Navarro, to be submitted.

[37] M. Wang, et al., Chinese Phys. C. 36, 1603 (2012).

[38] P. Möller, et al., Phys. Rev. C 79, 064304 (2009). 